\title{
Metabolic capability and in situ activity of microorganisms in an oil reservoir
}

\author{
Yi-Fan Liu ${ }^{1,2}$, Daniela Domingos Galzerani², Serge Maurice Mbadinga ${ }^{1,4}$, Livia S. Zaramela², Ji-Dong Gu, \\ Bo-Zhong $\mathrm{Mu}^{1,4^{*}}$ and Karsten Zengler $\mathrm{r}^{2,5^{*}}$
}

\begin{abstract}
Background: Microorganisms have long been associated with oxic and anoxic degradation of hydrocarbons in oil reservoirs and oil production facilities. While we can readily determine the abundance of microorganisms in the reservoir and study their activity in the laboratory, it has been challenging to resolve what microbes are actively participating in crude oil degradation in situ and to gain insight into what metabolic pathways they deploy.

Results: Here, we describe the metabolic potential and in situ activity of microbial communities obtained from the Jiangsu Oil Reservoir (China) by an integrated metagenomics and metatranscriptomics approach. Almost complete genome sequences obtained by differential binning highlight the distinct capability of different community members to degrade hydrocarbons under oxic or anoxic condition. Transcriptomic data delineate active members of the community and give insights that Acinetobacter species completely oxidize alkanes into carbon dioxide with the involvement of oxygen, and Archaeoglobus species mainly ferment alkanes to generate acetate which could be consumed by Methanosaeta species. Furthermore, nutritional requirements based on amino acid and vitamin auxotrophies suggest a complex network of interactions and dependencies among active community members that go beyond classical syntrophic exchanges; this network defines community composition and microbial ecology in oil reservoirs undergoing secondary recovery.
\end{abstract}

Conclusion: Our data expand current knowledge of the metabolic potential and role in hydrocarbon metabolism of individual members of thermophilic microbial communities from an oil reservoir. The study also reveals potential metabolic exchanges based on vitamin and amino acid auxotrophies indicating the presence of complex network of interactions between microbial taxa within the community.

Keywords: Auxotrophy, Hydrocarbon degradation, Metagenomics and metatranscriptomics, Microbial community, Oil reservoir

\section{Background}

Microorganisms have been detected in oil reservoirs, pipelines, and crude oil processing facilities for decades $[1,2]$. Their presence has long been associated with their metabolic capability of degrading crude oil hydrocarbons, under both oxic and anoxic conditions [3, 4]. While oil reservoirs are generally considered anoxic, molecular oxygen present in water injected into the reservoir during secondary recovery can play an

\footnotetext{
* Correspondence: bzmu@ecust.edu.cn; kzengler@ucsd.edu

'State Key Laboratory of Bioreactor Engineering and Institute of Applied

Chemistry, East China University of Science and Technology, 130 Meilong

Road, Xuhui District, Shanghai 200237, People's Republic of China

2Department of Pediatrics, University of California, San Diego, 9500 Gilman

Drive, La Jolla, San Diego, CA 92093-0760, USA

Full list of author information is available at the end of the article
}

important role as electron acceptor and can facilitate activation of otherwise chemically inert hydrocarbons [5]. Microorganisms associated to taxa consisting of predominately aerobic members have been detected and associated to the degradation of hydrocarbon in oil reservoirs undergoing secondary recovery [6].

Over the past decades, we have gained in depth knowledge, mainly through laboratory experiments, about how hydrocarbons are metabolized under oxic and anoxic conditions [7, 8]. Furthermore, recent advances into molecular biology and metabolomics have enabled elucidation of the metabolic potential of microbial communities existing in oil reservoirs [9]. Currently, however, we lack a deeper understanding of which members of the community are active in oil reservoirs and if these 
organisms are contributing to the degradation of crude oil in situ concurrently. Here, we analyze the aerobic and anaerobic microbial communities obtained from three wells of the Jiangsu Oil Reservoir (China) by an integrated metagenomic and metatranscriptomic approach to gain insight into the activity of specific microbes in situ. We annotate (almost complete) genomes from these samples and determine different activation mechanisms deployed during hydrocarbon degradation. Furthermore, we reveal nutritional requirements of oil reservoir microorganisms, such as amino acids and vitamins, hinting at a complex network of microbial interactions beyond syntrophy present in oil reservoirs.

\section{Results}

Metagenomic analysis of oil reservoir communities

Shotgun metagenomic sequencing of DNA obtained from wells W2-71, W9-18, and W15-5 in the Jiangsu Oil Reservoir (Jiangsu, China) generated 9,200,000, 10,900,000, and $\sim 7,700,000$ quality-controlled paired-end reads $(2 \times$ $75 \mathrm{bp}$ ), respectively (see Additional file 1: Table S1). In the remainder of this article, the three samples will be denoted as W2, W9 and W15. Sequence coverage was high for all three samples, ranging from 80 to $94 \%$ completion (see Additional file 2: Table S2) as estimated by Nonpareil [10]. Taxonomic classification from unassembled reads using MetaPhlAn2 and Metaxa2 yielded improved resolution over the assembled 16S rRNA gene sequences (Fig. 1). The biased estimation of taxonomic diversity is probably due to the low number of OTUs (13/17/16 (W2/W9/W15) clustered from 39/39/25 16S rRNA gene sequences) that were obtained from separately assembled contigs of the three samples (see Additional file 3: Table S3). As highlighted in Fig. 1, the most abundant bacterial orders in all three samples were Pseudomonadales, followed by Alteromonadales, Campylobacterales, and Thermodesulfobacteriales (Fig. 1). MetaPhlAn2 analysis revealed different proportions of Archaea compared to Metaxa2. This disaccord is expected since different biomarkers are used by these two tools to evaluate taxonomic information from metagenomes. Archaea sequences were dominated by members of the Methanosarcinales and Archaeoglobales. The overall microbial composition of three samples are similar except that members of Thermodesulfobacteriales and Thermoanaerobacterales were not detected in W2 samples, and relative low abundance of Methanosarcinales was found in W2 compared to other two samples. This is probably due to the lowest sequence coverage of W2 among the three samples, which affects abundance of minor members in microbial community, such as Archaeal members and minor community members of Bacteria. This is supported by a recent study in which similar microbial composition was reported in the same wells (W2-71 and W9-18) [11].
Metagenomic datasets were used to carry out differential coverage binning. A total of 44 genome bins (GBs) across all three samples were recovered (see Additional file 4: Table S4), representing $>95 \%$ of OTUs recovered (see Additional file 3: Table S3). Raw reads of the metagenome datasets were mapped to the GBs and normalized based on genome size to determine their relative abundance in each sample, and the result coincided with the result of taxonomic classification analysis above (see Additional file 4: Table S4). Seven high-quality genomes (completeness > 85\%, contamination <3\%) namely Acinetobacter-like Bin1, Marinobacter-like Bin13, Sulfurimonas-like Bin29, Thermodesulfobacterium-like Bin7, Archaeoglobus-like Bin9, Archaeoglobus-like Bin16, and Methanosaeta-like Bin39, which represent the most dominant members of Pseudomonadales, Alteromonadales, Campylobacterales, Thermodesulfobacteriales, Archaeoglobales and Methanosarcinales identified by assembled $16 \mathrm{~S}$ rRNA gene sequences (Fig. 1, Additional file 3: Table S3).

\section{Metabolic potential in genome bins}

To gain insight into the potential metabolism of microbes present in this oil reservoir, we analyzed the 44 GBs for genes and pathways associated with anaerobic degradation of hydrocarbons (see Additional file 5: Table S5). Archaeoglobus-like Bin9 contained the putative gene assA, which encodes the catalytic subunit of an alkylsuccinate synthase, an enzyme responsible for activation of hydrocarbons by fumarate addition [12] (Fig. 2a). Phylogenetic analysis of the putative assA gene showed a close relationship to assA gene in Archaeoglobus fulgidus (Accession number: AAB89800, see Additional file 6: Figure S1). A gene encoding alkylsuccinate synthase activating enzyme (assD) was also detected in Bin9 [13]. Genes for utilization of activated hydrocarbons were identified in Bin9, too. These genes include the putative CoA-synthetase/ligase (assK), alpha-methylacyl-CoA racemase, methylmalonylCoA mutase $(m c m L S)$, and methylmalonyl-CoA decarboxylase $(m c d)$ [14, 15]. Independent of the activation mechanism, genes involved in $\beta$-oxidation and subsequent utilization of acetyl-CoA are required for oxidation of hydrocarbons. Bin9 contained the complete $\beta$-oxidation pathway and an archaeal type Wood-Ljungdahl (WL) pathway (except the alpha and gamma subunit of formylMF dehydrogenase, i.e., $f w d A$ and $f w d C$ ). Bin9 also contained the complete pathway for dissimilatory sulfate reduction, namely sulfate adenylyltransferase (sat), adenylylsulfate reductase $(\operatorname{apr} A B)$, and the dissimilatory sulfide reductase $(d s r A B C)$. Similar pathways have been identified in the genome of the isolate Archaeoglobus fulgidus VC-16 [13]. In addition, five copies encoding ADP-forming acetyl-CoA synthetase $(a c d)$ were detected in $\operatorname{Bin} 9$, while strain VC-16 only contains three copies [13]. Despite the presence of $e h b Q$ (energy-converting hydrogenase B, 


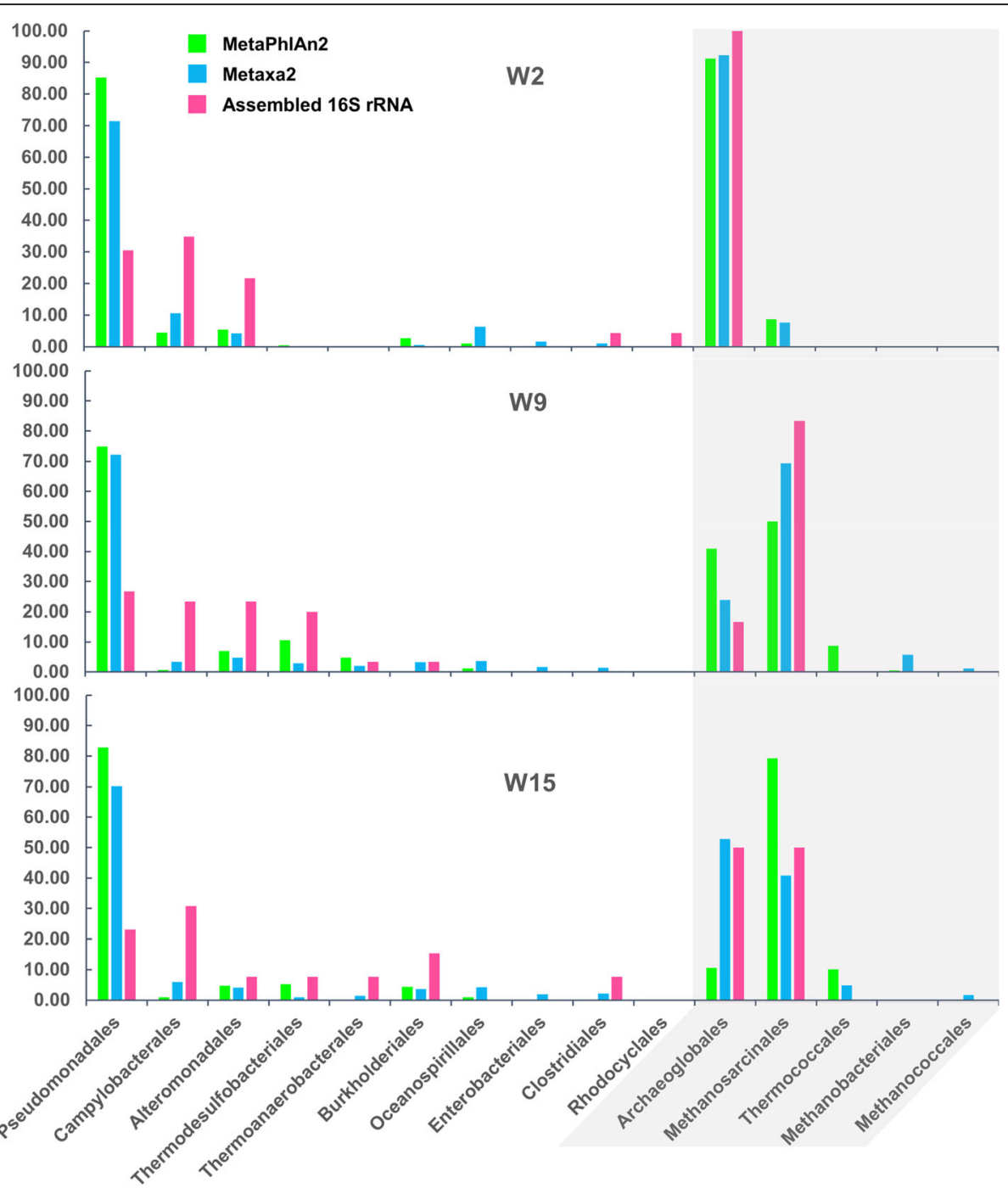

Fig. 1 Taxonomic analysis of metagenomes evaluated from unassembled reads by MetaPhIAn2 and Metaxa2 and from assembled 16S rRNA gene sequences. Bar graph is showing the percent abundance of the different members at order level, and only members with more than $0.1 \%$ abundance in either sample is showed here. Archaeal members are marked in gray. To get a better visualization, relative abundances of archaeal members are normalized to total number of archaeal members

subunit $\mathrm{Q})$ and $\operatorname{frcB}\left(\mathrm{F}_{420}\right.$-reducing hydrogenase, subunit beta), genes encoding for complete enzymes which are necessary for $\mathrm{H}_{2}$ or formate production, such as [FeFe] hydrogenase, $[\mathrm{NiFe}]$ hydrogenase or formate dehydrogenase, were absent in Bin9 [16].

In Methanosaeta-like Bin39, besides acetyl-coenzyme A synthase (acs) and acetyl-coenzyme A decarbonylase/synthase complex (codhABCDE) that are involved in acetoclastic methanogenesis, we also found genes involved in reduction of $\mathrm{CO}_{2}$ to methane, namely formyl methanofuran dehydrogenase (fwdABCDEFG), and also formyl methanofuran- $\mathrm{H}_{4} \mathrm{MPT}$ formyl-transferase (ftr), methenyl$\mathrm{H}_{4} \mathrm{MPT}$ cyclohydrolase $(m c h), \mathrm{F}_{420}$-dependent methylene$\mathrm{H}_{4} \mathrm{MPT}$ dehydrogenase $(m t d), \mathrm{F}_{420}$-dependent methylene $\mathrm{H}_{4} \mathrm{MPT}$ reductase (mer), tetrahydromethanopterin
( $\left.\mathrm{H}_{4} \mathrm{MPT}\right)$ - $S$-methyltransferase $(m t d)$, methyl-CoM reductase $(m c r A B C D G)$ and heterodisulfide reductase (hdrABCDG) (see Additional file 7: Figure S2). No formate dehydrogenase or hydrogenase was found in this bin.

Apart from genes associated to anaerobic hydrocarbon degradation, we also identified genes for aerobic hydrocarbon degradation from these wells. The integralmembrane alkane hydroxylase $(a l k B)$, which catalyze the terminal oxidation of medium/long chain $\left(>C_{6}\right)$ alkanes in the presence of oxygen [17], a rubredoxin, an electron transfer protein required by alkane hydroxylase [18], and a $\mathrm{NAD}(\mathrm{P}) \mathrm{H}$-dependent rubredoxin reductase $(\mathrm{rubB})$, were identified in the Acinetobacter-like Bin1 (Fig. 2b). In addition, a gene encoding for a Baeyer-Villiger monooxygenase $(\operatorname{alm} A)$, which is involved in subterminal 


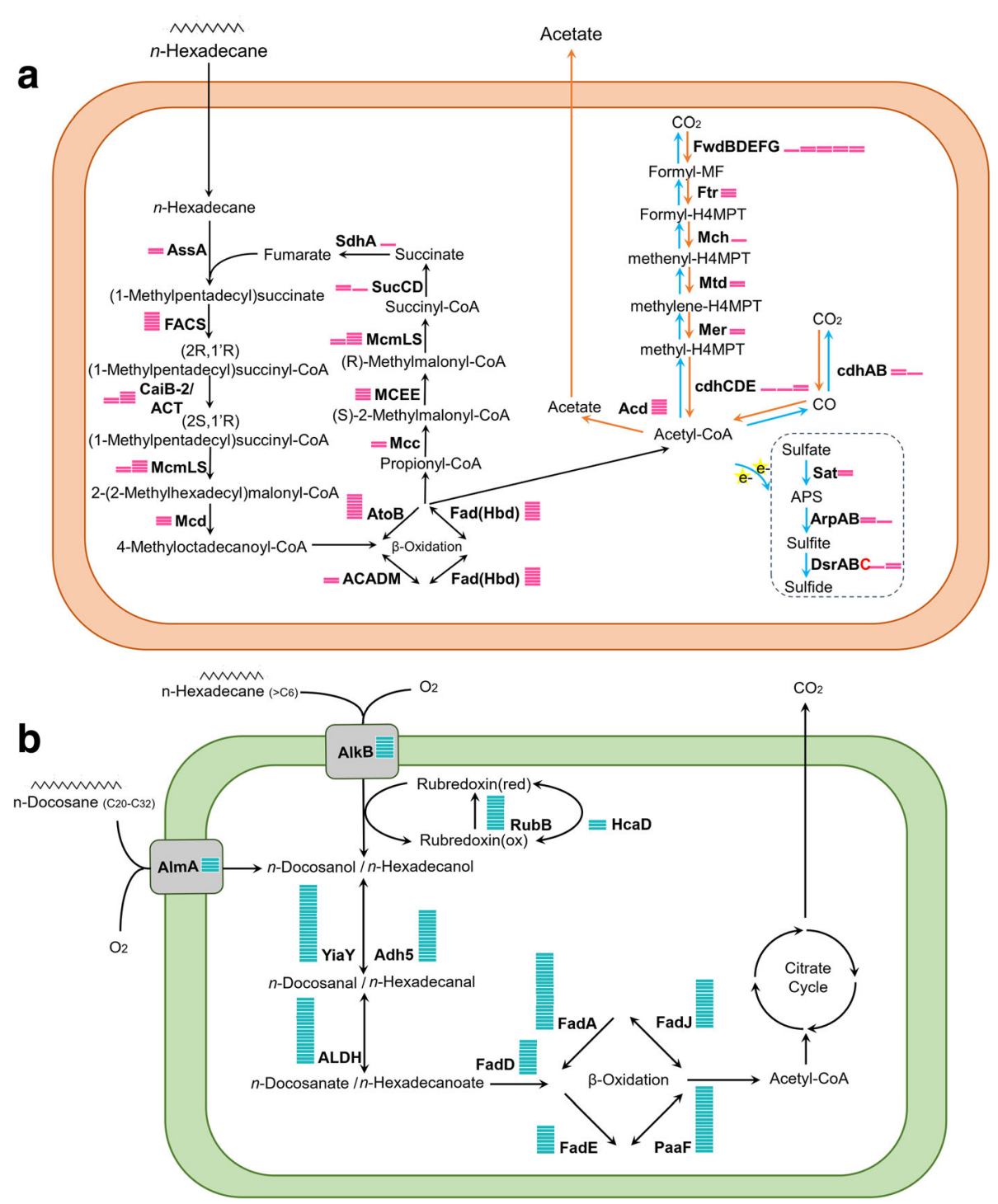

Fig. 2 Metabolic reconstruction of a putative model for $n$-hexadecane degradation in Bin9 (Archaeoglobus) and Bin1 (Acinetobacter). Expression level of each gene is represented by bars, one bar equaling a FPKM value of 10. Genes whose FPKM value equal 0 are marked in red. a Proposed anaerobic degradation of $n$-alkane by Bin9. Orange arrows indicate pathways that are associated with acetogenesis; blue arrows represent pathways that are associated with complete oxidation of $n$-alkanes coupled to sulfate reduction. $\mathbf{b}$ Proposed aerobic $n$-alkane degradation by Bin 1 based on genomic information

oxidation of long-chain alkanes $\left(\mathrm{C}_{20}-\mathrm{C}_{32}\right)$ [19], was identified in Bin1. Further analysis revealed that all genes necessary for processing of activated alkanes, such as alcohol dehydrogenase (adh), aldehyde dehydrogenase (aldh), and genes for $\beta$-oxidation, were also present in Bin1. alkB was also identified in Pseudomonas-like Bin19. However, likely due to low genome completeness of Bin19 (30.3\%), only adh and aldh were additionally detected in this GB.

\section{Transcriptional activity of community members}

Given the fact that microbial communities in three samples are similar (Fig. 1), RNA extracted from sample
W15 was chosen as a representative to study microbial metabolic activity in situ by using a metatranscriptomics approach [20] The cDNA sequencing generated $\sim 20$ million reads, and the sequence coverage estimated by Nonpareil [10] was very high (97\%) for W15. Metatranscriptomic reads were mapped to CDSs from previously assembled GBs, and the mapping rates of metatranscriptomic reads were normalized by the mapping rates of metagenomics reads from the same sample (W15) (Fig. 3). This analysis identified the most active microorganisms in situ. Pseudomonas-like Bin74 had the highest cDNA/DNA ratio (11.3), followed by Clostridia unclassified Bin22, Euryarcheota unclassified Bin54, Bacteria 


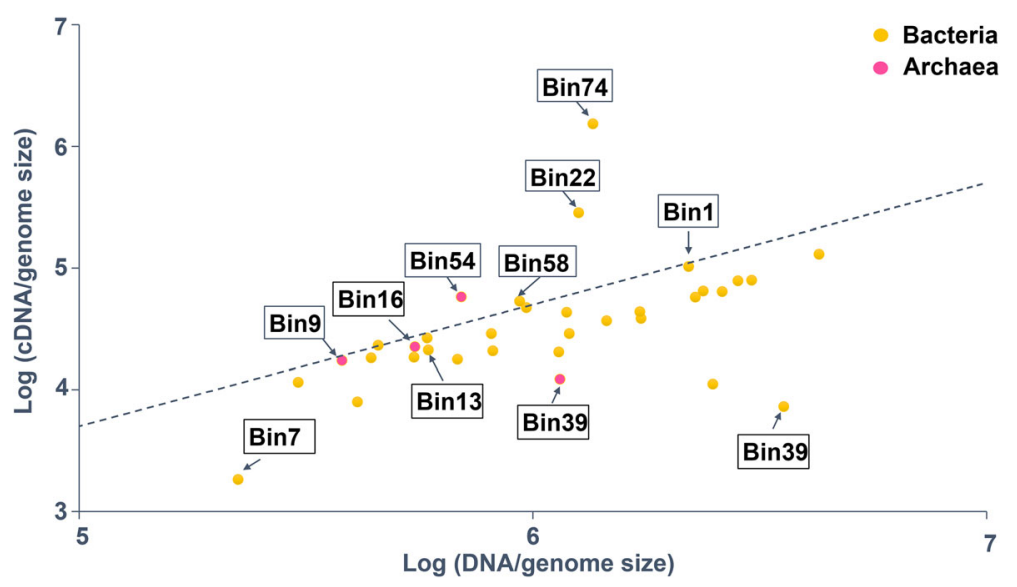

Fig. 3 DNA and CDNA mapping rate of GBs for sample W15. The GBs above the dashed line showed cDNA/DNA $>0.5$

unclassified Bin58, Alphaproteobacteria unclassified Bin72, and Betaproteobacteria unclassified Bin71 $(\geq 0.5)$. Archaeoglobus-like Bin9, Bin16 and Methanosaeta-like Bin39 exhibited a cDNA/DNA ratio of $0.45,0.1$, and 0.41 .

Metatranscriptomic sequence reads were mapped to co-assembled contigs to obtain genome-specific gene expression (see Additional file 8: Table S6). Genes with FPKM value $>10$, which ranked in the top $25 \%$ of all genes, were defined as highly transcribed (see Additional file 9: Figure S3). According to transcription analysis, the Archaeoglobus-like Bin9 transcribed the putative genes $a s s A$ and $a s s D$ at high level $(F P K M>10)$ (Fig. 2a). Genes involved in downstream pathways of alkane activation were also highly transcribed with FPKM values ranging from 30 to 70 in this genome. While genes for $\beta$-oxidation were highly transcribed (FPKM $>30$ ), methenyl- $\mathrm{H}_{4} \mathrm{MPT}$ cyclohydrolase gene $(m c h)$ of the WL pathway was transcribed at low level $($ FPKM $<10)$. Genes involved in sulfate reduction $(d s r A$ and $\operatorname{arp} B)$ were transcribed at a very low level $(\mathrm{FPKM}<5)$, and transcription of $d s r C$, the key gene for dissimilatory sulfate reduction [21], was below detection limit $(F P K M=0)$. By contrast, genes encoding the ADP-forming acetyl-CoA synthetase (acd) were highly expressed in Bin9 (total FPKM > 40).

Nearly all genes involved in acetoclastic methanogenesis and $\mathrm{CO}_{2}$-reductive methanogenesis, except for methyl-CoM reductase, subunit gamma $(m c r C)$ and subunit $\mathrm{B}$ and $\mathrm{F}$ of tetrahydromethanopterin methyltransferase $(m t r B, m t r F)$, were transcribed to different degree (see Additional file 8: Table S6).

Concurrently to anoxic alkane degradation, genes involved in oxic degradation of hydrocarbons were also highly transcribed (see Additional file 8: Table S6). Genes involved in aerobic alkane degradation from Acinetobacter-like Bin1 were highly expressed with FPKM value of $\operatorname{alm} A=62, \operatorname{alkB}=33, \operatorname{rub} A=28$ and $r u b B=103$ (Fig. 2b, also see Additional file 8: Table S6).
Genes involved in metabolizing activated alkanes, such as alcohol and aldehyde dehydrogenase and genes associated with $\beta$-oxidation, were also highly transcribed in this GB with FPKM values > 80. In comparison, the $a l k B$ gene of Pseudomonas-like Bin19 was transcribed at a significantly lower level (FPKM $=5.6)$, suggesting that Acinetobacter-like Bin1 contributes more to aerobic alkane oxidation in well W15.

\section{Amino acid and vitamin auxotrophies}

Auxotrophies and exchange of amino acids and vitamins have been shown to reinforce interactions in a syntrophic alkane-oxidizing community in the laboratory $[20,22]$. We therefore analyzed the seven nearly complete GBs (> 85\% complete), i.e., Archaeoglobus Bin9 and Bin16, Thermodesulfobacterium Bin7, Methanosaeta Bin39, Sulfurimonas Bin29, Marinobacter Bin13, and Acinetobacter Bin1 from the oil reservoir for amino acid and vitamin auxotrophies (Fig. 4). In specific, the four most abundant anaerobic microorganisms had the capacity to synthesize all essential amino acids but histidine, and all vitamins but B7. A similar result was observed for the three most complete GBs of aerobic community members. While the three aerobic microbes where not able to synthesize vitamins B6 and B12, amino acid auxotrophies were much less abundant in these organisms, with Sulfurimonas and Marinobacter being able to synthesize all amino acids (Fig. 5).

\section{Discussion}

Microbial utilization of hydrocarbons from crude oil has long been known to occur under both oxic and anoxic conditions. While pristine oil reservoirs were considered mainly anoxic, insertion of injection water during secondary oil recovery introduces molecular oxygen to these environments, generating suitable conditions for both aerobic and anaerobic microbial communities to thrive. 


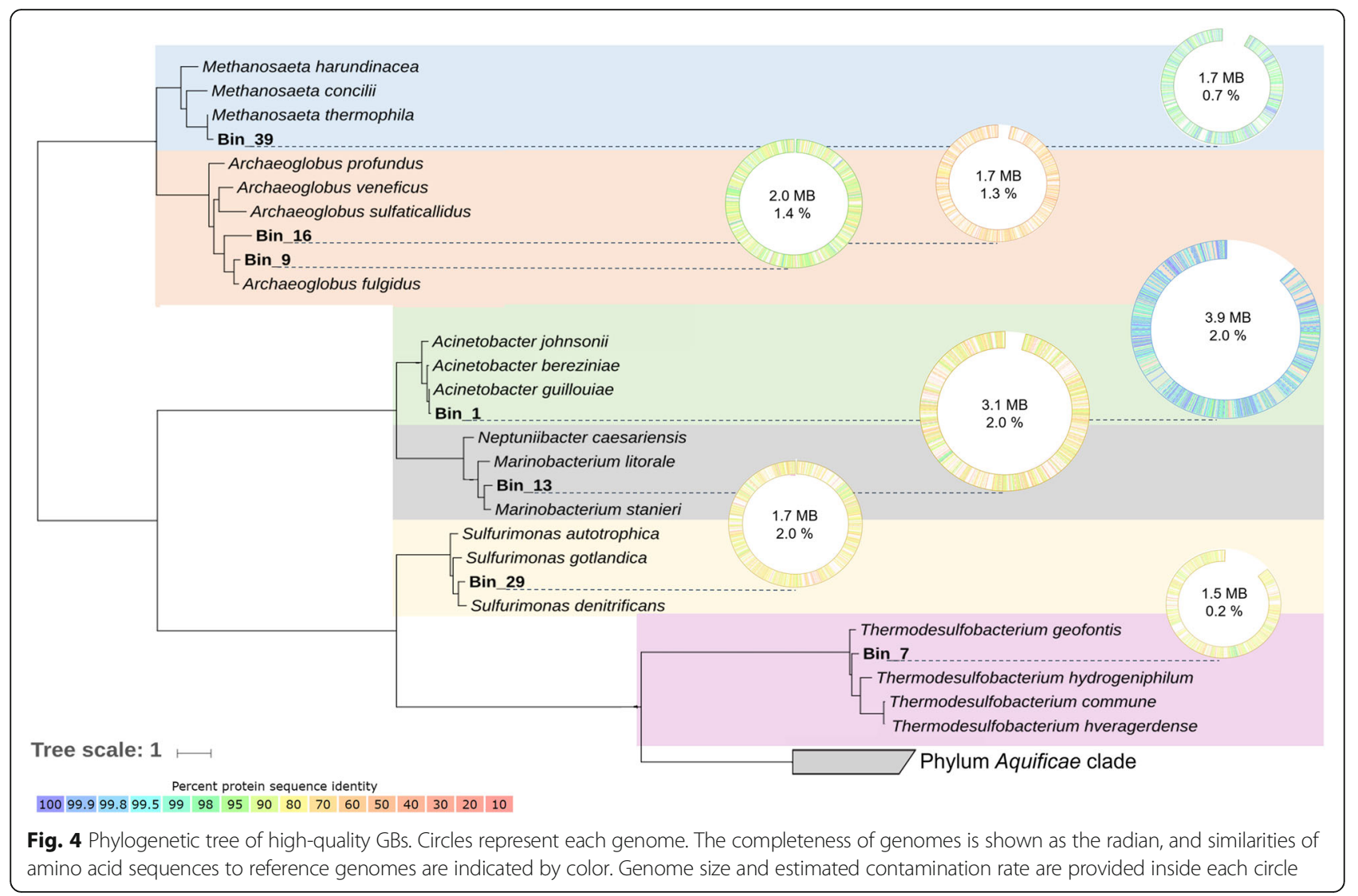

Dominant taxa identified by shotgun metagenomics in this study were also the most abundant microorganisms detected by an amplicon metagenomics approach [11]. Microorganisms associated to taxa generally consisting of obligate aerobic bacteria, such as Acinetobacter and Marinobacter, were abundant in all three wells from the Jiangsu oil reservoir. These aerobic bacteria are believed to participate in the degradation of hydrocarbons in the oil reservoir using molecular oxygen [6, 23]. Facultative anaerobes, Pseudomonas species have been frequently detected in oil reservoirs [24, 25], and some members of them are capable of degrading aromatic hydrocarbons or alkanes [26, 27]. Although anaerobic archaea, such as Archaeoglobus, as well as Methanosaeta were present at lower abundance than aerobic bacteria, they were found with relatively high transcript level in W15 sample, suggesting their important roles in oil reservoir. $A$. fulgidus strain VC-16, a representative of the genus Archaeoglobus, isolated from marine hydrothermal systems can degrade $n$-alkanes $\left(C_{10}-C_{21}\right)$ with thiosulfate or sulfate as terminal electron acceptor [28]. The mapping rate of metatranscriptomic reads implies concurrent activity of aerobic microorganisms, such as Pseudomonas and Acinetobacter, and anaerobic microorganisms such as Archaeoglobus and Methanosaeta in this well.

\section{Acetogenic fermentation of alkanes}

While the exact mechanism of hydrocarbon activation in A. fulgidus VC-16 or the Archaeoglobus-like Bin9 is still unclear at the time, it is assumed that a glycyl radical enzyme (AssA) is responsible for the activation in A. fulgidus $\mathrm{VC}-16$ [28]. A gene sequence annotated as ass $A$ has been identified in Bin9. While Bin9 is capable of sulfate respiration, the presence of $a c d$ and genes for $\beta$-oxidation in Bin9 suggests that this microorganism can ferment organic compounds, like fatty acids or $n$-alkanes, when electron acceptors are depleted [29]. Moreover, gene expression indicates that most cells are fermenting under in situ conditions using the WL pathway (Fig. 2a). In this process, acetate formation from acetyl-CoA couples with reductive acetogenesis from $\mathrm{CO}_{2}$, and energy was conserved using Acd during hydrocarbon degradation [29]. According to thermodynamic calculation as proposed by Dolfing et al. [30], pH and acetate concentration found in the Jiangsu oil reservoir would also support incomplete oxidation of alkanes to acetate in these wells (see Additional file 10: Table S7 and Additional file 11: Figure S4) [30, 31]. Low levels of sulfate and absence of sulfide in well W15, as well as lack of expression of $d s r C$ further support a fermentative rather than respiratory life style of the alkanedegrading Archaeoglobus-like Bin9 (see Additional file 12: Table S8 and full details are in Additional file 13: Text S1) 


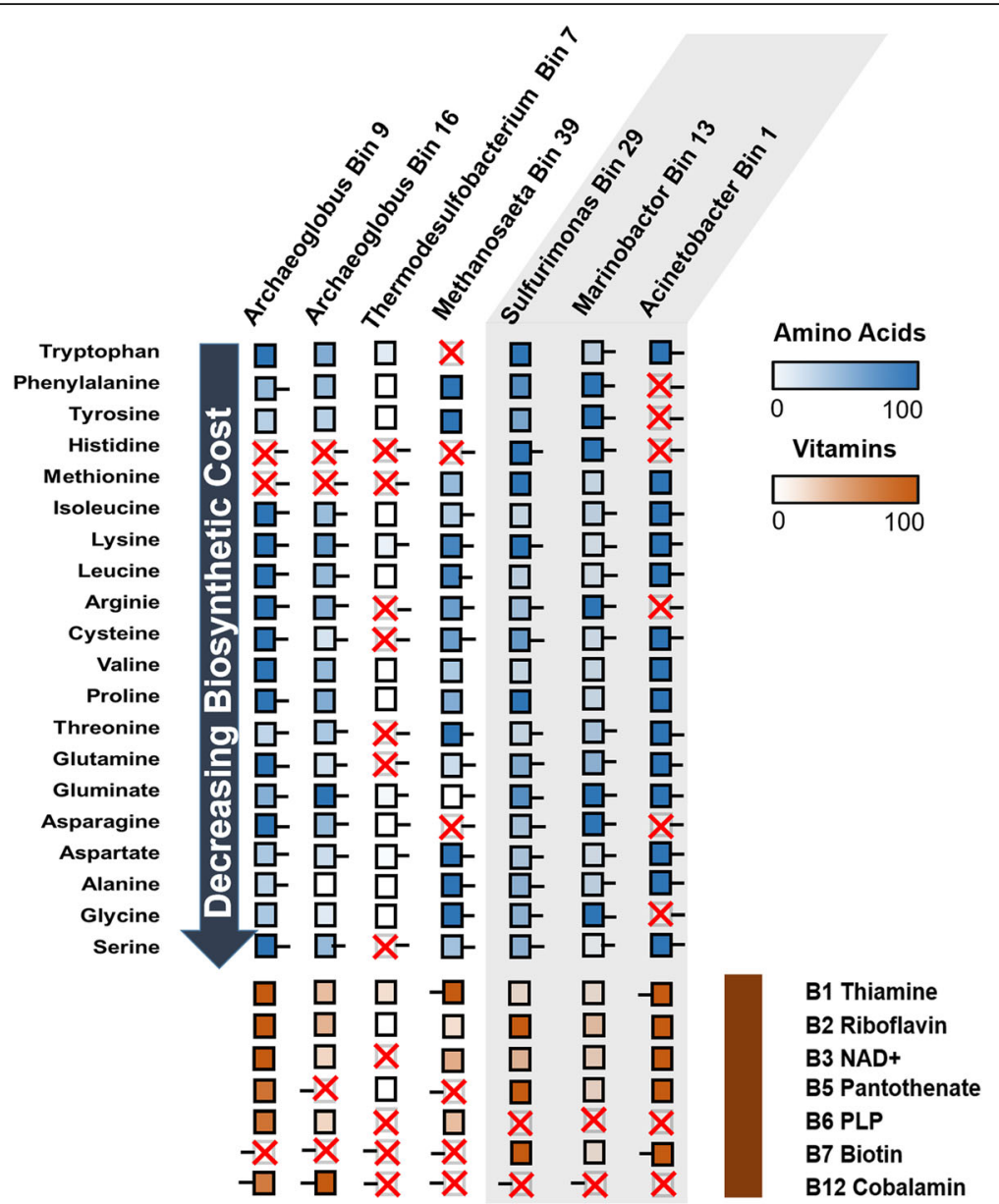

Fig. 5 Specific amino acid auxotrophies and vitamin auxotrophies present in high-quality GBs. The auxotrophy of specific compound was confirmed if more than one gene was absent in the synthetic pathway, or overall transcriptional level of pathway was low compared to genomes with complete pathway in which only one gene was absent. Aerobic species are covered in gray. Amino acids are in depicted in blue and vitamins in brown. Amino acids have been ranked according to biosynthetic cost (arrow). A colored square denotes that a species can synthesize an amino acid. The intensity of each color (based on the scale) represents the relative expression of the synthesis pathways, with darker/more intense color indicating higher expression. Auxotrophies are marked by a red cross. A black line next to the box represents the presence of a transporter. A transport system is not required for vitamins $B 2$ and $B 3$

in the reservoir. Based on genomic and transcriptomic evidence, we speculated that Archaeoglobus-like Bin9 ferments hydrocarbons to acetate in situ, which is consumed by other microorganisms.

\section{Potential methanogenic activity via direct interspecies electron transfer}

The presence of Methanosaeta in the samples hints at methanogenic degradation of hydrocarbons [20]. Although methane was not measured in the samples, methanogenic enrichment cultures could be obtained from these samples. The expression analysis indicated active acetoclastic methanogenesis and $\mathrm{CO}_{2}$-reductive methanogenesis in well W15. The absence of formate dehydrogenase or hydrogenase in this bin was consistent with studies on other Methanosaeta species, suggesting direct interspecies electron transfer (DIET) when $\mathrm{CO} 2$ serves as electron acceptor for these methanogens [32, 33]. However, one should bear in mind that Bin39 represents an incomplete genome sequence and while formate dehydrogenase and hydrogenase are absent in other Methanosaeta genomes, these genes could be present in Bin39. The high expression of fwdG $(\mathrm{FPKM}>80)$ also suggested $\mathrm{CO}_{2}$-reduction to occur via DIET in addition to acetoclastic methanogenesis, as describe before [20, 32, 34].

\section{Complete oxidation of alkanes}

The detection of alkane hydroxylase, together with genes encoding for rubredoxin and rubredoxin reductase in Acinetobacter-like Bin1, indicate the ability of this microorganism to oxidize alkanes aerobically. The cooccurrence of different types of alkane hydroxylases (alkB and almA) implied that Bin1 could potentially utilize different strategies for degrading alkanes with 
variable chain length $[17,19]$. A putative pathway for complete oxidation of $n$-alkanes by $\operatorname{Bin} 1$ was reconstructed (Fig. 2b) [35]. The high levels of transcription of genes associated to hydrocarbon degradation suggest that Acinetobacter actively participates in the oxic degradation of hydrocarbons in the oil reservoir.

\section{Amino acid and vitamin auxotrophies}

None of the seven GBs had all genes required for synthesizing all amino acids and vitamins, hinting at complex interdependencies among microorganisms in this oil reservoir (Fig. 5), similar to what has been described for communities of both long- and short-chain hydrocarbon-degrading communities from enrichment cultures [20, 22]. The absence of microbial members that synthesize specific vitamins and amino acids in the aerobic and anaerobic communities suggested that additional partners are needed for these communities to thrive. According to the result, intertwined dependencies based on amino acid auxotrophy thus seem to be more prevalent in the anoxic community. Interestingly, amino acids auxotrophies in the aerobic microorganisms were not as dominant as in the anaerobic microorganisms. Reduced energy yields for microorganisms growing with hydrocarbons under anoxic conditions compared to oxic conditions might be responsible for dominant amino acid auxotrophies observed here and in a previous study based on laboratory communities [20, 22]. Individual aerobic microbes from this oil reservoir are capable for complete hydrocarbon oxidation, diminishing their requirement for metabolic interaction. However, all amino acids and vitamins could be synthesized when both aerobes and anaerobes were taken as a unit.

The prevalence of amino acid and vitamin auxotrophies within the key members of the anaerobic community in the Jiangsu oil reservoir hint at a highly intertwined network of interactions. A similar network has been described in a methanogenic alkane- and fatty aciddegrading communities where very few members were capable of histidine synthesis [20]. While histidine auxotrophies were most prevalent among community members investigated here, auxotrophies of Methanosaeta-like Bin39 were different to what was previously reported for a Methanosaeta strain in syntrophic communities propagated in the laboratory [20], indicating that interactions are dependent on individual strains and can vary based on community composition. Further investigations of amino acid auxotrophies are needed to reveal if this is a hallmark of syntrophic communities degrading hydrocarbons anaerobically. While this study demonstrated the concurrent activity of different communities, it is currently unknown how these communities are organized in time and space. Thus, studies are needed that account for the spatial organization of members of the aerobic and anaerobic communities, e.g., by SIP or NanoSIMS.

\section{Conclusions}

While aerobic and anaerobic microorganisms have simultaneously been identified from oil reservoirs undergoing secondary recovery, it has often been difficult to delineate what role these organisms are playing for the microbial ecology of the oil reservoir. The main reason is that injection waters carry large number of microbes that can contribute significantly to abundance profiles from these reservoirs but are not actively participating in the degradation of oil hydrocarbons. Here, we elucidated the metabolic potential of individual members of thermophilic microbial communities from an oil reservoir in Jiangsu, China, by metagenomics and determined their role in hydrocarbon metabolism in situ by metatranscriptomics. We identified key members of the aerobic and anaerobic community performing oxic or anoxic hydrocarbon degradation and determined that a large fraction of microorganisms detected by omics approaches were mostly inactive, likely originating from the injection water. Along with potential metabolic exchanges, we identified prevalent auxotrophies in members of this thermophilic community, hinting at a complex network of interactions. While both aerobic and anaerobic communities are active in the oil reservoir at the same time, sampling at high spatial resolution is required to delineate potential interactions and exchanges between these two communities.

\section{Methods}

\section{Samples of production water}

Production water samples for DNA and RNA isolation were collected on March, 19, 2015, from three different wells, i.e., W2-71, W9-18 and W15-5, in the Jiangsu Oil Reservoir (Jiangsu, China). Forty liters of production water was collected for each sample for DNA extraction, and another $40 \mathrm{~L}$ of production water for RNA extraction was stabilized using a 10\% (vol/vol) stop solution (95\% ethanol, 5\% TRIzol (Life Technology)) [34]. All samples were kept on ice and transported to the laboratory within $4 \mathrm{~h}$ and immediately centrifuged at $12,000 \times g$ at $4{ }^{\circ} \mathrm{C}$ for $10 \mathrm{~min}$ upon arrival in the lab. After removal of supernatant, pellets were kept at $-80^{\circ} \mathrm{C}$ and processed within 2 weeks.

\section{Metagenomic and metatranscriptomic sequencing}

DNA and RNA were extracted from pellets using the PowerMicrobiome $^{\mathrm{Tm}}$ RNA Isolation Kit (MO BIO). To enrich messenger RNA (mRNA), ribosomal RNA was depleted from total RNA using Ribo-Zero rRNA Removal Kit (Bacteria) (Illumina ${ }^{\oplus}$ ) according to the manufacturer's instructions. Double-strand cDNA was synthesized as described before [34]. Sequencing libraries for both metagenomic and metatranscriptomic were prepared using the 
Nextera XT DNA Library Prep Kit (Illumina"). The quality of the libraries was checked using a Bioanalyzer High Sensitivity Chip (Agilent), and the libraries were quantified by Qubit dsDNA HS Assay. The metagenomics libraries were sequenced on an Illumina Miseq using 150-cycle kit $(2 \times$ $75 \mathrm{pb}$ paired-end). The metatranscriptomic libraries were sequenced on the same platform using a 50-cycle kit.

\section{Taxonomic assessment of unassembled metagenomic reads}

Filtered metagenomic reads were used for taxonomic assessment by screening for SSU rRNAs with Metaxa2 v2.1.3 [36] and for phylogenetic marker genes using MetaPhlAn2 v2.7.0 [37].

\section{Metagenome assembly and analysis}

The metagenomic raw reads were examined using FastQC tool (http://www.bioinformatics.babraham.ac.uk/projects/ fastqc/), and low-quality sequences were trimmed using PRINSEQ v0.20.4 (parameters: 'trim_tail_right', 5; trim_tail_left', 5; entropy threshold, 50; minimum read length of 70 base; maximum replication, 6; maximum 'n's, 0) [38]. The level of coverage of each metagenomic dataset was estimated using Nonpareil with default parameters [10]. The trimmed reads were independently assembled de novo using SPAdes v3.7.0 with the following parameters '-meta $-\mathrm{k} 33,45,61,71$ '. The contigs of each metagenome were annotated using MG-RAST online server [39]. To generate a reference dataset for further additional analysis, trimmed reads from the three metagenomes were combined and co-assembled using SPAdes.

Ribosomal genes were identified using two complementary methods. In summary, (1) the dominant species (based on reconstructed near-full-length $16 \mathrm{~S}$ rRNA gene) of each metagenome were identified using EMIRGE with default parameters [40] and (2) rRNA_hmm [41] was used to search assembled contigs containing $16 \mathrm{~S}$ rRNA sequences. 16S rRNA sequences > 240 bp identified by EMIRGE and rRNA_hmm were combined for further analysis. Redundant $16 \mathrm{~S}$ rRNA genes were manually evaluated by blasting [42] each EMIRGE-reconstructed sequence to rRNA_hmm-found sequences. If the alignment showed $100 \%$ of identity, the rRNA_hmm sequence was discarded. 16S rRNAs chimeric sequences were identified and deleted using DECIPHER [43]. In order to generate representative OTUs, non-chimeric sequences were clustered using CD-HIT v4.6 on $97 \%$ of similarity [44]. Ribosomal Database Project (RDP) were used to assign the OTUs to their corresponding taxonomy [45].

\section{Screening for functional genes}

Metagenomic reads from three samples were combined to get better assembly and greater coverage of the genomic bins [46] (full details in Additional file 14: Text S2). Co-assembled contigs were examined for the presence of genes associated with oxic and anoxic hydrocarbon-degrading pathways [47, 48]. Hidden Markov Models (HMMs) for translated sequences were constructed in-house using HMMER (v3.1b1) [49]. To construct HMMs, amino-acid sequences for hydrocarbon-degrading pathways proteins were obtained from GenBank and aligned using MAFFT. Multiple alignments were manually curated using AliViewv 1.18 [50]. Curated alignments were used as an input to HMMER to create HMMs. The HMMs were used to search for hydrocarbon-degrading proteins among the translated genes obtained from the co-assembled metagenome using Prodigal v2.6 [51]. If the number of sequences were not enough to build a HMM, individual protein sequences were searched against the metagenome translated genes using BLASTP with $E$-value $<\mathrm{e}^{-10}$ [42]. All hits were manually curated using NCBI NR database and false positives were discarded.

\section{Differential binning of metagenomic reads}

Co-assembled contigs were used for differential binning [52]. In brief, individual trimmed reads were mapped back to the contigs file, and the resulting bam files were used as input into GroopM with default parameters (v0.3.4) [52]. De-contamination of retrieved genome bins (GBs) was carried out in ProDeGe [53]. Bonafide GBs were uploaded to RAST [54] for annotation. All GBs were checked for completeness and contamination using checkM v1.0.5 [55].

\section{Genome analysis}

PhyloPhlAn v0.99 [56] was used to reconstruct the phylogenetic tree of all GBs based on the protein prediction results from Prodigal v2.6 [51]. Additionally, we used BLAST [42] to compare the 16S rRNA gene annotated in each bin. Amino acid synthesis pathway, vitamin synthesis pathways, and vitamins transport system in near-complete GBs were curated manually using primary literature and the KEGG database [57] as previously described [20]. Metatranscriptomic dataset of W15 was used in addition to help curate amino acid and vitamin synthesis pathways. For biosynthetic pathways in which only one gene was missing, we used metatranscriptomic information to evaluate expression levels of the pathway. If the overall transcription level was similar or higher than in GBs that harbor a complete pathway, this pathway was considered complete.

\section{Metatranscriptome analysis}

Metatranscriptomic raw reads were trimmed by quality using Prinseq (parameters were identical to the metagenome analysis), and trimmed reads were mapped to GB's coding sequences (CDS) using Bowtie2 [58] with default settings. Transcription level of each recovered 
GB was evaluated by computing the cDNA/DNA abundance ratio. Transcription level of individual genes in each GB was determined by mapping metatranscriptomic reads to co-assembled contigs using Bowtie2 [58] with default parameters. eXpress v1.5.1 [59] was used to calculate FPKM (Fragments Per Kilobase of transcript per Million) values, and the genes whose FPKM values rank in top 25th percentile were defined as actively expressed.

\section{Additional files}

Additional file 1: Table S1. Summary of assembled contigs. (DOCX 14 $\mathrm{kb})$

Additional file 2: Table S2. Estimate of metagenomic sequencing coverage. (DOCX $21 \mathrm{~kb}$ )

Additional file 3: Table S3. Taxonomic classification of assembled $16 \mathrm{~S}$ rRNA gene sequences from metagenomes of W2, W9 and W15. (DOCX $31 \mathrm{~kb})$

Additional file 4: Table S4. Information of genome bins (separate file). The completeness, contamination and strain heterogeneity of GBs were evaluated by CheckM. The columns for taxonomy were splited into three, within which the 'ProDeGe Tax' column recorded the information from ProDeGe server based on BlastP search, the 'Rast_Closet_Spp.' column recorded information from RAST server using its 'View closest neighbors' function and the 'Phylophlan_Assignment' column recorded the lineage output using Phylophlan. (XLSX $18 \mathrm{~kb}$ )

Additional file 5: Table S5. Information of GBs studied in context. (DOCX $17 \mathrm{~kb}$ )

Additional file 6: Figure S1. Phylogenetic tree of amino acid sequences of assA genes. (PNG $287 \mathrm{~kb}$ )

Additional file 7: Figure S2. Proposed Syntrophic model for (a) Methanosaeta and (b) Archaeoglobus. The expression level of each gene is represented by bars (pink and blue), with one bar representing FPKM value of 10. Genes for which not transcripts could be mapped (FPKM value $=0$ ) are marked in red. (TIFF $1778 \mathrm{~kb}$ )

Additional file 8: Table S6. Annotation and FPKM value of hydrocarbon degradation and methanogenesis related genes in GBs (separate file). Annotation of studied GBs was done by a combined effort of RAST server and KEGG database. (XLSX $429 \mathrm{~kb}$ )

Additional file 9: Figure S3. Cumulative distribution plot of genes under certain FPKM value. Scatter plot of accumulated number of genes in co-assembled scaffolds under certain FPKM value based on the metatranscriptome W15. Genes with FPKM value less than 10 accounted for $75 \%$ of total genes, therefore genes with FPKM above 10 were defined as actively expressed genes. (TIFF $396 \mathrm{~kb}$ )

Additional file 10: Table S7. Thermodynamic constraint of acetate concentration on oxidation of hexadecane to acetate. (DOCX $15 \mathrm{~kb}$ )

Additional file 11: Figure S4. Effect of $\mathrm{pH}$ on the range of acetate concentrations when $\mathrm{n}$-alkanes (i.e. hexadecane as representative for calculation) are oxidized to acetate as sole fermentation product and coupled to acetoclastic methanogenesis. The green line represents the oxidation of hexadecane to acetate; red line represents the conversion of acetate to $\mathrm{CH}_{4}$. Blue dots represent acetate concentration and $\mathrm{pH}$ values present in wells W2, W9 and W15. The arrows indicate conditions under which the process becomes exergonic. The highlighted zone (light blue) indicates a window of opportunity where oxidization of hexadecane to acetate coupled to acetoclastic methanogenesis is thermodynamically favorable. The figure was modified from [30]. (TIFF $682 \mathrm{~kb}$ )

Additional file 12: Table S8. Geochemical characterization of production water from three wells from the Jiangsu oil field, China. ( ${ }^{*} \mathrm{ND}$, not detected.) (DOCX $15 \mathrm{~kb})$
Additional file 13: Text S1. Supplementary results and discussion. (DOCX $17 \mathrm{~kb}$ )

Additional file 14: Text S2. Supplementary materials and methods. (DOCX 29 kb)

\section{Acknowledgements}

The authors are grateful to the management of the Jiangsu Oil Reservoir for sampling support.

\section{Funding}

This work was supported by the National Natural Science Foundation of China (Grant No. 41530318, 41373070) to YFL, SMM, and BZM and the NSFC/ RGC Joint Research Fund (No. 41161160560) to JDG.

China Scholarship Council (CSC) funded Yi-Fan Liu's doctoral scholarship in a joint-PhD program with the University of California, San Diego. Additionally, Daniela Domingos Galzerani was supported by CNPq under the Brazilian Scientific Mobility Program, Ciências sem Fronteiras (No 201011/2014-0). This study is in part based on work supported by the US Department of Energy (DOE), Office of Science, Office of Biological \& Environmental Research Awards DE-SC0012586 and the Frontiers of Innovation Scholars Program at UCSD.

Availability of data and materials

Sequence data were deposited to NCBI under the SRA accession numbers SRX2648638 to SRX2648643.

\section{Authors' contributions}

YFL performed the fieldwork, geochemical measurements of water samples, and analysis of metagenomics and metatranscriptomic datasets. DDG extracted the DNA and RNA from samples and constructed sequencing libraries. YFL, SMM, JDG, LSZ, and KZ wrote the manuscript. BZM and KZ oversaw this project. All authors read and approved the final manuscript.

Ethics approval and consent to participate

Not applicable

\section{Consent for publication}

Not applicable

Competing interests

The authors declare that they have no competing interests.

\section{Publisher's Note}

Springer Nature remains neutral with regard to jurisdictional claims in published maps and institutional affiliations.

\section{Author details}

${ }^{1}$ State Key Laboratory of Bioreactor Engineering and Institute of Applied Chemistry, East China University of Science and Technology, 130 Meilong Road, Xuhui District, Shanghai 200237, People's Republic of China.

${ }^{2}$ Department of Pediatrics, University of California, San Diego, 9500 Gilman Drive, La Jolla, San Diego, CA 92093-0760, USA. ${ }^{3}$ School of Biological Sciences, The University of Hong Kong, Pokfulam Road, Hong Kong, People's Republic of China. ${ }^{4}$ Shanghai Collaborative Innovation Center for Biomanufacturing Technology, Shanghai 200237, People's Republic of China. ${ }^{5}$ Center for Microbiome Innovation, University of California, San Diego, 9500 Gilman Drive, La Jolla, San Diego, CA 92093-0436, USA.

Received: 22 August 2017 Accepted: 19 December 2017

Published online: 05 January 2018

\section{References}

1. Stevenson BS, Drilling HS, Lawson PA, Duncan KE, Parisi VA, Suflita JM. Microbial communities in bulk fluids and biofilms of an oil facility have similar composition but different structure. Environ Microbiol. 2011;13: 1078-90.

2. Duncan KE, Gieg LM, Parisi VA, Tanner RS, Tringe SG, Bristow J, et al. Biocorrosive thermophilic microbial communities in Alaskan North Slope oil facilities. Environ Sci Technol. 2009;43:7977-84. 
3. Head IM, Jones DM, Larter SR. Biological activity in the deep subsurface and the origin of heavy oil. Nature. 2003;426:344-52.

4. Aitken CM, Jones DM, Larter SR. Anaerobic hydrocarbon biodegradation in deep subsurface oil reservoirs. Nature. 2004;431:291-4.

5. Head IM, Gray ND, Larter SR. Life in the slow lane; biogeochemistry of biodegraded petroleum containing reservoirs and implications for energy recovery and carbon management. Front Microbiol. 2014;5:1-23.

6. An D, Caffrey SM, Soh J, Agrawal A, Brown D, Budwill K, et al. Metagenomics of hydrocarbon resource environments indicates aerobic taxa and genes to be unexpectedly common. Environ Sci Technol. 2013;47:10708-17.

7. Zengler K, Richnow HH, Rosselló-Mora R, Michaelis W, Widdel F. Methane formation from long-chain alkanes by anaerobic microorganisms. Nature. 1999:401:266-9.

8. Heider J, Spormann AM, Beller HR, Widdel F. Anaerobic bacterial metabolism of hydrocarbons. FEMS Microbiol Rev. 1998;22:459-73.

9. Bian X-Y, Mbadinga SM, Liu Y-F, Yang S-Z, Liu J-F, Ye R-Q, et al. Insights into the anaerobic biodegradation pathway of $n$-Alkanes in oil reservoirs by detection of signature metabolites. Sci Rep. 2015;5:9801. Nature Publishing Group. Available from: http://www.nature.com/srep/2015/150513/ srep09801/full/srep09801.html.

10. Rodriguez-R LM, Konstantinidis KT. Nonpareil: a redundancy-based approach to assess the level of coverage in metagenomic datasets. Bioinformatics. 2014;30:629-35.

11. Li X-X, Liu J-F, Zhou L, Mbadinga SM, Yang S-Z, Gu J-D, et al. Diversity and composition of sulfate-reducing microbial communities based on genomic DNA and RNA transcription in production water of high temperature and corrosive oil reservoir. Front Microbiol. 2017:8:1-17. Available from: https://www.frontiersin.org/articles/10.3389/fmicb.2017.01011/full.

12. Callaghan AV, Wawrik B, Ní Chadhain SM, Young LY, Zylstra GJ. Anaerobic alkane-degrading strain AK-01 contains two alkylsuccinate synthase genes. Biochem Biophys Res Commun. 2008:366:142-8.

13. Klenk HP, White O, Ketchum KA. The complete genome sequence of the hyperthermophilic, sulphate-reducing archaeon Archaeoglobus fulgidus. Nature. 1997;390:364-70.

14. Callaghan a V, Morris BEL, Pereira I a C, Mclnerney MJ, Austin RN, Groves JT, et al. The genome sequence of Desulfatibacillum alkenivorans AK-01: a blueprint for anaerobic alkane oxidation. Environ Microbiol. 2012;14:101-13.

15. Jarling R, Sadeghi M, Drozdowska M, Lahme S, Buckel W, Rabus R, et al. Stereochemical investigations reveal the mechanism of the bacterial activation of $n$-alkanes without oxygen. Angewandte Chemie - International Edition. 2012;51:1334-8.

16. Worm P, Stams AJM, Cheng X, Plugge CM. Growth- and substratedependent transcription of formate dehydrogenase and hydrogenase coding genes in Syntrophobacter fumaroxidans and Methanospirillum hungatei. Microbiology. 2011;157:280-9.

17. Ratajczak A, Geißdörfer W, Hillen W. Alkane hydroxylase from Acinetobacter sp. strain ADP1 is encoded by alkM and belongs to a new family of bacterial integral-membrane hydrocarbon hydroxylases. Appl Environ Microbiol. 1998;64:1175-9.

18. Geißdörfer W, Kok RG, Ratajczak A, Hellingwerf KJ, Hillen W. The genes rubA and rubB for Alkane degradation in Acinetobacter sp. strain ADP1 are in an Operon with estB, encoding an Esterase, and oxyR. J Bacteriol. 1999;181: 4292-8. Available from: http://jb.asm.org/content/181/14/4292.abstract.

19. Minerdi D, Zgrablic I, Sadeghi SJ, Gilardi G. Identification of a novel Baeyer-Villiger monooxygenase from Acinetobacter radioresistens: close relationship to the Mycobacterium tuberculosis prodrug activator EtaA. Microb Biotechnol. 2012;5: 700-16. Available from: http://doi.wiley.com/10.1111/j.1751-7915.2012.00356.x

20. Embree M, Liu JK, Al-bassam MM, Zengler K. Networks of energetic and metabolic interactions define dynamics in microbial communities. Proc Natl Acad Sci U S A. 2015:112:15450-55

21. Santos AA, Venceslau SS, Grein F, Leavitt WD, Dahl C, Johnston DT, et al. A protein trisulfide couples dissimilatory sulfate reduction to energy conservation. Science. 2015;350:1541-5. Available from: http://www sciencemag.org/content/350/6267/1541.full.

22. Hubalek V, Buck M, Tan B, Foght J, Wendeberg A, Berry D, et al. Vitamin and amino acid auxotrophy in anaerobic consortia operating under methanogenic conditions. mSystems. 2017;2:e00038-17. Available from: http://msystems.asm.org/lookup/doi/10.1128/mSystems.00038-17.

23. Gutierrez T, Singleton DR, Berry D, Yang T, Aitken MD, Teske A. Hydrocarbon-degrading bacteria enriched by the Deepwater Horizon oil spill identified by cultivation and DNA-SIP. ISME J. 2013;7:2091-104.
Nature Publishing Group. Available from: http://www.nature.com/ doifinder/10.1038/ismej.2013.98.

24. Gao P, Tian H, Wang Y, Li Y, Li Y, Xie J, et al. Spatial isolation and environmental factors drive distinct bacterial and archaeal communities in different types of petroleum reservoirs in China. Sci Rep. 2016;6: 20174. Nature Publishing Group. Available from: http://www.nature.com/ articles/srep20174

25. Wang LY, Ke WJ, Sun XB, Liu JF, Gu JD, Mu BZ. Comparison of bacterial community in aqueous and oil phases of water-flooded petroleum reservoirs using pyrosequencing and clone library approaches. Appl Microbiol Biotechnol. 2014;98:4209-21.

26. Cladera AM, Bennasar A, Barceló M, Lalucat J, García-Valdés E. Comparative genetic diversity of Pseudomonas stutzeri genomovars, clonal structure, and phylogeny of the species. J Bacteriol. 2004;186:5239-48.

27. Mehboob F, Junca H, Schraa G, Stams AJM. Growth of Pseudomonas chloritidismutans AW-1T on n-alkanes with chlorate as electron acceptor. Appl Microbiol Biotechnol. 2009;83:739-47.

28. Khelifi N, Amin Ali O, Roche P, Grossi V, Brochier-Armanet C, Valette O, et al. Anaerobic oxidation of long-chain n-alkanes by the hyperthermophilic sulfate-reducing archaeon, Archaeoglobus fulgidus. ISME J. 2014;8:3057-60. Available from: http://www.nature.com/doifinder/10.1038/ismej.2014.58.

29. Musfeldt M, Schönheit P. Novel type of ADP-forming acetyl coenzyme A synthetase in hyperthermophilic Archaea: Heterologous expression and characterization of isoenzymes from the sulfate reducer Archaeoglobus fulgidus and the methanogen Methanococcus jannaschii. J Bacteriol. 2002; 184:636-44.

30. Dolfing J, Larter SR, Head IM. Thermodynamic constraints on methanogenic crude oil biodegradation. ISME J. 2008;2:442-52. Available from: https://doi. org/10.1038/ismej.2007.111.

31. Henstra AM, Dijkema C, Stams AJM. Archaeoglobus fulgidus couples CO oxidation to sulfate reduction and acetogenesis with transient formate accumulation. Environ Microbiol. 2007;9:1836-41.

32. Rotaru A-E, Shrestha PM, Liu F, Shrestha M, Shrestha D, Embree M, et al. A new model for electron flow during anaerobic digestion: direct interspeciesa electron transfer to Methanosaeta for the reduction of carbon dioxide to methane. Energy Environ Sci. 2014;7:408. Available from: http:// xlink.rsc.org/?DOI=c3ee42189a.

33. Holmes DE, Shrestha PM, Walker DJ, Dang Y, Nevin KP, Woodard TL, et al. Metatranscriptomic evidence for direct interspecies electron transfer between Geobacter and Methanothrix species in Methanogenic Rice Paddy soils; 2017. p. 1-11.

34. Embree M, Nagarajan H, Movahedi N, Chitsaz H, Zengler K. Single-cell genome and metatranscriptome sequencing reveal metabolic interactions of an alkane-degrading methanogenic community. ISME J. 2014;8:757-67. Nature Publishing Group. Available from: http://www.nature.com/doifinder/ 10.1038/ismej.2013.187.

35. Dombrowski N, Donaho JA, Gutierrez T, Seitz KW, Teske AP, Baker BJ. Reconstructing metabolic pathways of hydrocarbon-degrading bacteria from the Deepwater Horizon oil spill. Nature Microbiology. 2016;1:1-8. Nature Publishing Group. Available from: https://doi.org/10.1038/nmicrobiol.2016.57.

36. Bengtsson-Palme J, Hartmann M, Eriksson KM, Pal C, Thorell K, Larsson DGJ, et al. metaxa2: improved identification and taxonomic classification of small and large subunit rRNA in metagenomic data. Mol Ecol Resour. 2015;15:1403-14.

37. Segata N, Waldron L, Ballarini A, Narasimhan V, Jousson O, Huttenhower C. Metagenomic microbial community profiling using unique clade-specific marker genes. Nat Methods. 2012;9:811-4.

38. Schmieder R, Edwards R. Quality control and preprocessing of metagenomic datasets. Bioinformatics. 2011;27:863-4.

39. Meyer F, Paarmann D, D'Souza M, et al. The metagenomics RAST server-a public resource for the automatic phylo- genetic and functional analysis of metagenomes. BMC bioinformatics. 2008;9:386.

40. Miller CS, Baker BJ, Thomas BC, Singer SW, Banfield JF. EMIRGE: reconstruction of full-length ribosomal genes from microbial community short read sequencing data. Genome Biol. 2011;12:R44. Available from: http://genomebiology.com/2011/12/5/R44.

41. Huang $Y$, Gilna P, Li W. Identification of ribosomal RNA genes in metagenomic fragments. Bioinformatics. 2009;25:1338-40.

42. Altschul SF, Madden TL, Schäffer AA, Zhang J, Zhang Z, Miller W, et al Gapped BLAST and PS I-BLAST: a new generation of protein database search programs. Nucleic Acids Res. 1997;25:3389-402. Available from: https://doi.org/10.1093/nar/25.17.3389. 
43. Wright ES, Yilmaz LS, Noguera DR. DECIPHER, a search-based approach to chimera identification for 16S rRNA sequences. Appl Environ Microbiol. 2012;78:717-25.

44. Fu L, Niu B, Zhu Z, Wu S, Li W. CD-HIT: accelerated for clustering the nextgeneration sequencing data. Bioinformatics. 2012;28:3150-2.

45. Cole JR, Wang Q, Fish JA, Chai B, McGarrell DM, Sun Y, et al. Ribosomal database project: data and tools for high throughput rRNA analysis. Nucleic Acids Res. 2014;42:633-42.

46. Baker BJ, Lazar CS, Teske AP, Dick GJ. Genomic resolution of linkages in carbon, nitrogen, and sulfur cycling among widespread estuary sediment bacteria. Microbiome. 2015:3:1-12.

47. Tan B, Jane Fowler S, Laban NA, Dong X, Sensen CW, Foght J, et al. Comparative analysis of metagenomes from three methanogenic hydrocarbon-degrading enrichment cultures with 41 environmental samples. ISME J. 2015;9:2028-45. Nature Publishing Group. Available from: https://doi.org/10.1038/ismej.2015.22.

48. Foght J. Anaerobic biodegradation of aromatic hydrocarbons: pathways and prospects. J Mol Microbiol Biotechnol. 2008;15:93-120.

49. Eddy S. Profile hidden Markov models. Bioinformatics. 1998;14:755-63. Available from: http://bioinformatics.oxfordjournals.org/content/14/9/755.short

50. Larsson A. Aliview: a fast and lightweight alignment viewer and editor for large datasets. Bioinformatics. 2014:30:3276-8.

51. Hyatt D, Chen G-L, Locascio PF, Land ML, Larimer FW, Hauser LJ, et al. BMC bioinformatics. 2010;11:119. Available from: http://www.pubmedcentral.nih gov/articlerender.fcgi?artid=2848648\&tool=pmcentrez\&rendertype=abstract.

52. Imelfort M, Parks D, Woodcroft BJ, Dennis P, Hugenholtz P, Tyson GW. GroopM: an automated tool for the recovery of population genomes from related metagenomes. PeerJ. 2014;2:e603. Available from: https://peerj.com/ articles/603.

53. Tennessen K, Andersen E, Clingenpeel S, Rinke C, Lundberg DS, Han J, et al. ProDeGe: a computational protocol for fully automated decontamination of genomes. ISME J. 2015;10:1-4. Nature Publishing Group. Available from: https://www.nature.com/articles/ismej2015100.

54. Aziz RK, Bartels D, Best AA, DeJongh M, Disz T, Edwards RA, et al. The RAST Server: rapid annotations using subsystems technology. BMC Genomics. 2008;9:75. Available from: http://bmcgenomics.biomedcentral.com/articles/ 10.1186/1471-2164-9-75

55. Parks DH, Imelfort M, Skennerton CT, Hugenholtz P, Tyson GW. CheckM: assessing the quality of microbial genomes recovered from isolates, single cells, and metagenomes. Genome Res. 2015;25:1043-55.

56. Segata N, Börnigen D, Morgan XC, Huttenhower C. PhyloPhIAn is a new method for improved phylogenetic and taxonomic placement of microbes. Nat Commun. 2013;4:2304. Available from: http://www.pubmedcentral.nih. gov/articlerender.fcgi?artid=3760377\&tool=pmcentrez\&rendertype=abstract.

57. Ogata H, Goto S, Sato K, Fujibuchi W, Bono H, Kanehisa M. KEGG: Kyoto encyclopedia of genes and genomes. Nucleic Acids Res. 1999;27:29-34.

58. Langmead B, Salzberg SL. Fast gapped-read alignment with bowtie 2. Nat Methods. 2012;9:357-9.

59. Roberts A, Pachter L. Streaming fragment assignment for real-time analysis of sequencing experiments. Nat Methods. 2013;10:71-3. Available from: https://doi.org/10.1038/nmeth.2251.

\section{Submit your next manuscript to BioMed Central and we will help you at every step:}

- We accept pre-submission inquiries

- Our selector tool helps you to find the most relevant journal

- We provide round the clock customer support

- Convenient online submission

- Thorough peer review

- Inclusion in PubMed and all major indexing services

- Maximum visibility for your research

Submit your manuscript at www.biomedcentral.com/submit

) Biomed Central 